\title{
POLITICA E PREVISIONE TECNOLOGICA: UN CODICILLO A DANIEL BELL
}

\author{
di Giovanni Sartori
}

Senza un criterio di decifrazione, senza una chiave esplicativa, tanto il passato come il futuro sono soltanto zone di buio. La ricerca di questo «decifratore» appartiene, tradizionalmente, a quel capitolo della filosofia che viene detto «filosofia della storia ». Ma per quanto si dica cosí, è chiaro che una volta fatta la scelta del criterio esplicativo questo criterio deve valere indifferentemente tanto per il passato come per il futuro, tanto per la retrospezione come per la previsione, tanto all'indietro come in avanti. In pratica, tuttavia, chi cerca di decifrare il futuro si trova meglio con talune chiavi esplicative che non con altre. Non è affatto una coincidenza che il futurologo opti, il piú delle volte, per un tipo di spiegazione tecnologica e tecnostrutturale.

Se le «cause ultime » del cambiamento si trovano nella tecnologia e nell'babitat tecnico, allora le nostre congetture e profezie poggiano su un terreno relativamente solido, e cioè su dati « duri ». Per contro, quanto piú ci lasciamo alle spalle le «determinazioni oggettive », e quanto piú il nostro principio di decifrazione si richiama a «fattori soggettivi », di altrettanto il futurologo si sente a disagio. Il che sta anche per dire che il sociologo e l'economista che lavorano su dati «duri » non hanno difficoltà a passare alla previsione, laddove il passaggio è piú arduo per lo storico e il politologo che lavorano su dati «soffici ».

Di qui la differenza tra previsioni tecnologiche che poggiano su fondamenta economico-sociologiche, da un lato, e, dall'altro lato, una incertezza predittiva che si richiama ad elementi di prova di tipo storico-culturale. I recenti scritti di Daniel Bell che anticipano il suo volume su $L$ a società post-industriale ${ }^{1}$ rappresentano, a mio

Questo scritto è ricavato dalla relazione orale al Convegno di Zurigo del giugno 1970 organizzato da Daniel Bell e Ralph Dabrendorf sul tema "La società postindustriale ». Le relazioni al detto Convegno sono state pubblicate su Survey, n. 78, Winter 1971.

1 Vedili nei segg. voll. coll.: Eli Ginzberg (ed.), Technology and Social Change, New York, Columbia University Press, 1965; E. \& E. Hutchings (ed.), Scientific Progress and Human Values, New York, American Elsevier, 1966; Bertram M. Gross (ed.), A Great Society?, New York, Basic Books, 1968; Daniel Bell 
avviso, quanto di meglio sia stato scritto in chiave tecnologica, nel senso piú ampio e sottile del concetto. Pur fondando le sue profezie su proiezioni di tipo tecnologico, Bell è ben consapevole dei limiti di queste proiezioni. Nella relazione su La società post-industriale: tecnocrazia e politica, Bell si preoccupa espressamente di come la concezione tecnocratica - che si richiama ad una particolare accezione della razionalità - si rapporta alla politica.

Nelle sue parole, «la visione tecnocratica è destinata a diffondersi. Ma non ne consegue che $\mathrm{i}$ tecnocrati diverranno una classe dominante, cosí come resta aperto il problema di come la visione tecnocratica potrà venire contestata $»$. Nel seguito del discorso Bell rileva che « nei prossimi decenni l'arena della politica diventerà piú decisiva che mai, per due motivi fondamentali: le decisioni cruciali... sono prese dai governi assai piú che tramite il mercato; e diventano sempre piú numerosi i gruppi che avanzano e cercano di stabilire le proprie rivendicazioni sulla società passando attraverso l'ordinamento politico ». E Bell conclude con questa osservazione: la politica «precede sempre la razionalità, e spesso sconvolge la razionalità $~^{2}$. Non c'è dubbio, dunque, sul fatto che Bell avverte come la previsione tecnologica sia, di fronte alla politica, di corta gittata.

C'è modo di aggirare questa difficoltà? Bell sembra pensare, o sperare, di sí. La sua idea, mi par di capire, è questa: che il fattore culturale, la «cultura», sia un elemento intermedio, e in qualche misura fissante tra tecnologia e politica. Il suo ragionamento sembra essere, tra le righe, che se fossimo in condizione di spiegare le diversità culturali saremmo anche in condizione di controllare meglio le «incertezze » della politica. Difatti nelle note introduttive al convegno di Zurigo Bell si chiede se «i moduli di valore e le tradizioni culturali non valgano a spiegare le variazioni tra società piú e meglio del fattore economico e tecnologico». Senonché Bell avverte che mentre «la cultura si internazionalizza e i valori (quantomeno i valori espressi dalla scienza e dal sapere) diventano sempre piú universali $»$, nulla di simile sembra accadere nel settore politico: in politica le differenze restano. $E$ dunque Bell finisce per tornare al quesito: «se le differenze politiche sono la fonte di divergenza tra le varie società, come spiegare queste differenze? ». Il problema che tormenta il nostro autore si può dunque riassumere cosí. L'interesse di Bell si appunta, in ultima analisi, sul rapporto tra tecnologia e politica; ma egli cerca di vedere se la poli-

(ed.), Toward the Year 2000, Boston, Houghton Mifflin, American Academy of Arts and Sciences, 1968. Vedi inoltre Daniel Bell, Notes on the Post-Industrial Society, in "The Public Interest», n. 6 (Winter 1967) e n. 7 (Spring 1967); The Post-Industrial Society: Technocracy and Politics, in «Survey», n. 78 (仓े la relazione al Convegno di Zurigo alla quale si riferisce specificamente questa nota); The Post-Industrial Society: The Evolution of an Idea, in «Survey », n. 79, Spring 1971.

2 In « Survey », n. 78, cit. 
tica possa essere inserita e fissata nella «cultura», e ciò legata a qualcosa di meno sfuggente e incostante di quanto non risulti la politica di per sé presa.

Tecnologia, cultura e comunicazione di massa.

Cominciamo da come l'imperativo tecnologico si rapporta con la cultura e con la diversità culturale. $\mathrm{Ci}$ imbattiamo subito, qui, nel problema che chiamavo all'inizio di filosofia della storia, e cioè di scelta della chiave esplicativa. Una scelta che ci colloca su sponde opposte. È vero che cerchiamo di mantenere un piede su entrambe le sponde. Bell, per esempio, attribuisce gran peso a quella che denomina «cultura avversativa». Nondimeno è chiaro che tutto il suo discorso sulla società post-industriale dipende da indicatori e predittori «oggettivi» di tipo tecno-scientifico e tecno-strutturale. Per lui la politica e il porsi di una «cultura avversativa» costituiscono una variabile interveniente, e anzi una variabile perturbante. Laddove dall'altra sponda - quella della « crisi culturale » di Michel Crozier o della «rivolta psicologica» di cui parla Stanley Hoffman - il fattore culturale risulta la variabile indipendente.

Non ne consegue che lo studioso che adotta il filo esplicativo culturale (o delle idee) non possa accettare il discorso di Bell. Chi dà il primato alla forza delle idee non ha alcun bisogno di contestare gli argomenti sull'imperativo industriale, sulle « inevitabilità » della scienza e sulla mentalità tecnocratica. Siamo tutti persuasi del fatto che la rivoluzione tecnologica modifica profondamente la struttura occupazionale, e che questa trasformazione trasforma, a sua volta, la struttura sociale e, in definitiva, il nostro modo di vivere e di concepire la vita. Ma tutti questi argomenti non risultano conclusivi per chi pone il fattore esplicativo nella cultura e nelle idee. Nella misura in cui non crediamo nelle «determinazioni oggettive », nella stessa misura l'obiezione è: quando si è detto tutto sull'ambiente, resta da dire dell'uomo. L'uomo ha la capacità di « determinare le determinazioni $»$, e comunque non reagisce meccanicamente all'babitat. E qui ci ritroviamo schierati su sponde opposte.

In che misura le due posizioni esplicative - tecnostrutturale e culturale - possono essere avvicinate e proporzionate l'una con l'altra? Per rispondere occorre rendere piú maneggevole l'aggrovigliata matassa delle tante cose che sono sottintese dalla diżione «tecnologia». La domanda è: nel complesso dei molteplici fattori tecnologici, quali sono gli elementi o fattori che investono piú frontalmente, e che incidono piú profondamente, sull'uomo? Conviene distinguere, a questo effetto, tra 1) fattori indiretti di cambiamento, e cioè quei cambiamenti che cambiano l'uomo tramite l'ambiente, e 2) fattori diretti di cambiamento, nel senso che costituiscono 
quell'ultimo anello di una catena tecnologica, che arriva direttamente all'uomo. In particolare conviene distinguere tra tecnologia della produzione e una vera e propria «tecnologia dell'uomo», una tecnologia che finisce per generare un « uomo nuovo »: nuovo si badi bene - nel senso che non somiglia ai suoi predecessori, non nel senso di essere un uomo migliore, rigenerato.

Vi sono almeno due ragioni per spostare l'attenzione dalla tecnologia della produzione alla tecnologia dell'uomo. La prima è che su questo terreno l'argomento tecnologico e l'argomento culturale si congiungono. La seconda ragione è la semplicità. Se la nostra messa a fuoco si concentra sul destino dell'uomo, i fattori da considerare possono essere ridotti a due: l'accelerazione storica da un lato, e la rivoluzione delle comunicazioni di massa dall'altro. Per il primo rispetto il problema è la vertigine della velocità; per il secondo è il bombardamento del messaggio.

L'accelerazione della storia non è un fatto nuovo, nel senso che diventa sensibile già con la rivoluzione francese e, per un altro rispetto, con la rivoluzione romantica. Da allora, comunque, la «macchina del tempo » è diventata sempre piú veloce; e sicuramente esiste un limite di velocità non visibile, al quale l'uomo non riesce ad adattarsi. Oggi come oggi l'individuo si sente « superato » prima dell'esaurirsi del suo ciclo biologico. Non solo i mestieri dei padri non sono piú trasmissibili ai figli, ma siamo continuamente soggetti alla necessità del recyclage. La velocità del cambiamento $\mathrm{e}$ dell'innovazione è tale che la vita di una generazione è sempre piú segnata da discontinuità traumatizzanti. Ma non occorre insistere su un problema $\mathrm{i}$ cui termini sono noti da tempo. Occorre invece mettere a fuoco meglio l'altro problema: il bombardamento del messaggio.

La tecnologia delle comunicazioni di massa comporta la «vittoria del cannone ». Lungo tutto il corso della storia è esistita la gara tra arma e armatura, tra proiettile e corazza, tra carro d'assalto e linea Maginot. Per millenni la vicenda è stata alterna: talvolta prevalendo lo strumento di offesa, talaltra quello di difesa. Oramai è chiaro che il cannone ha vinto, sia in guerra (la Bomba, il Germe) che in pace: l'offensiva del messaggio soverchia le nostre possibilità di difesa. L'uomo come animale mentale non è mai stato tanto esposto, tanto vulnerabile, quanto lo è oggi. Il potenziale della tecnologia delle comunicazioni di massa è tale da travolgere - se davvero utilizzato sino in fondo - i nostri meccanismi di difesa mentale. Chi sostiene il contrario non vede lontano, e soprattutto è afflitto da una visione «parrocchiale». Certo: oggi, nel mondo libero, il consumatore del messaggio si può difendere, reagisce con il disinteresse, e anzi «retroagisce» sull'emittente del messaggio. Analogamente, il mondo libero stabilisce un parametro e un punto di riferimento per il mondo che libero non è. Il che 
non toglie che il potenziale della tecnologia del messaggio è quello che è, e sanziona la vittoria definitiva del cannone sulla corazza.

Il secondo punto da fermare è che la tecnologia delle comunicazioni di massa sta ristrutturando le linee di convergenza e di divergenza tra gli agglomerati umani. Vale a dire che le comunicazioni di massa stanno erodendo il mondo longitudinale (e cioè storicamente ancorato e diversificato) delle nazioni, al quale si sovrappone e sottentra sempre piú un mondo orizzontale e sincronico di «movimento di massa ». Lo Stato nazionale è sempre meno una unità di analisi significativa. I sistemi sociali diventano sempre piú transnazionali non solo in sede economica, ma anche in chiave antropologica, e cioè in ragione di quel che Eisenstadt chiama la « distruzione delle nicchie » e che Bell denomina la «perdita di spazio isolante ».

Se le comunicazioni di massa distruggono le nicchie, eliminano gli spazi isolanti e spianano le barriere verticali poste dalla storia e dalle tradizioni culturali, quel che ne emerge è un mondo orizzontale le cui unità organizzative e di analisi sono $\mathrm{l}^{\prime}$ «immagine $\mathrm{e}$ il messaggio ». Grossissimo modo possiamo ipotizzare tre grandi insiemi di reti di comunicazione, e per essi tre mondi: a) un mondo di comunicazioni di massa policentriche ed erratiche; b) dei mondi totalitari e unicentrici di indottrinamento di massa (URSS, Cina, e relativi satelliti); c) un mondo residuale e embrionale di comunicazioni di massa imperniato sui transistors - su messaggi senza immagini - che investe le aree di povertà cronica dell'Africa, della America Latina e del Sud-Est Asiatico. Una piú precisa raffigurazione di questi «aggregati comunicanti» potrà essere data dalla struttura aperta o chiusa delle varie reti comunicative, e misurata da indici di densità radio-televisiva. Comunque sia, il punto è che il baricentro del nostro «habitat simbolico » si è radicalmente modificato, spostandosi dalla parola scritta e parlata all'immagine visiva; che la centralità dell'immagine visiva segna sempre piú profondamente la nostra esistenza; e che l'abitante della società tecnologica sta diventando sempre piú uni-dimensionale nel senso che viene sempre piú plasmato lungo la sola dimensione di un istantaneo «tempo presente».

La mia congettura è dunque che l'impatto di linee vaganti di comunicazioni orizzontali «libera »l'uomo nuovo dal suo passato, dalle sue radici culturali, e da qualsiasi ancoraggio preesistente, sia per il bene come per il male. In questo senso gli uomini stanno sicuramente «convergendo », e cioè diventando piú monotoni, piú simili, attraverso le frontiere nazionali (ma non, si badi, attraverso tutto il mondo: poiché nuove frontiere sono create dalle reti di comunicazione alle quali appartengono le singole comunità comunicanti). La convergenza di cui sopra non crea amicizia e convergenza politica tra $\mathrm{i}$ popoli, cosí come non cancella, anche se può 
modificare, le aree di ostilità e di affinità ideologica. È una convergenza che semmai aumenta l'imprevedibilità. Perché un mondo ancorato a, e quindi disancorato da, flussi vaganti ed erratici di comunicazione di massa è un mondo che fluttua secondo «movimenti lampo » di opinione e di passione. $E$ questo per due rispetti. Per un primo rispetto, quanto piú comanda la « voce del messaggio », tanto piú è possibile riscaldare, manipolare e mobilitare masse di uomini ora in una direzione ora nell'altra. Per il secondo rispetto gli «effetti dimostrativi» e gli effetti di contagio e di rinforzo delle comunicazioni audio-visive producono di volta in volta accensioni o esplosioni mal prevedibili che escono di controllo.

In sintesi, il crescendo di accelerazione storica combinato con i fenomeni di rinforzo e di contagio istantaneo prodotti dalle comunicazioni di massa scalza non solo le fondamenta storiche della previsione - la previsione come proiezione del passato nel futuro ma anche le previsioni fondate sull'imperativo razionale e sulla razionalità dell'imperativo tecnologico. E se - come vado sostenendo - la rivoluzione delle comunicazioni di massa è la rivoluzione decisiva, ne segue che l'interrogativo che piú condiziona il nostro futuro è: chi controllerà in che modo il bombardamento del messaggio? Il che ci riporta alla politica.

\section{Difficoltà e necessità della previsione politica.}

A prima vista, e da parecchi punti di vista, la politica è l'elemento meno trattabile, o piú ostico, del problema. In primo luogo, non esiste un «imperativo politico » nello stesso senso in cui esiste un «imperativo tecnologico ». Nel senso pieno dell'espressione l'imperativo politico investe la Buona Vita, o la Buona Società; e non siamo affatto d'accordo, in concreto, sui valori e sulle priorità della Città Ideale. Se tutti dichiarano di volere la democrazia, tutti la vogliono diversa. Come è invalso l'uso di dire, si tratta di un unanimismo fittizio.

Tuttavia è lecito parlare di imperativo politico in un significato piú limitato dell'espressione, e cioè accantonando la dimensione e la disputa sui valori. In questo significato piú limitato l'imperativo politico è dato da quei meccanismi politici che sono « imperativamente » richiesti quale vis à vis tecnico, o strumentale, da una società che postula un altissimo grado di interdipendenza, di sincronizzazione, per ciò stesso di fragilità tecnologica: la società che Brzezinski dice «tecnotronica » ${ }^{3}$. La città tecnotronica è un

3 Cfr. Z. Brzezinski, Between Two Ages: America's Role in the Technetronic Era, New York, Viking Press, 1970. L'a. dichiara di preferire «tecnotronica » a 《 post-industriale » in quanto la sua etichetta mette in evidenza 《 gli impulsi principali del cambiamento»(p. 9). A me piace soprattutto perché 
mondo ad altissima precisione, un mondo regolato ad orologeria, e piú esattamente regolato dagli elaboratori elettronici. Quale è l'imperativo politico che ne emerge o che lo fiancheggia? E davvero, questo, un quesito « intrattabile »? Non mi sembra. La futurologia politica non è in auge soprattutto perché richiede il coraggio di dire verità impopolari. Certo è che il mondo tecnotronico non sarà un mondo ad autogestione assembleare, senza « repressione $»$, senza comandi, e ancor meno senza Stato.

$\mathrm{Ma}$ torniamo al quesito ad boc: chi controllerà, in che modo, gli strumenti di comunicazione di massa? Una domanda che si inquadra nel quesito di fondo sulle sorti e sulle possibilità di sopravvivenza della démocrazia.

Di quale democrazia? Quali che siano le nostre idee normative su cosa la democrazia dovrebbe essere, quel che effettivamente e indiscutibilmente riesce ad essere - nella teoria e nella prassi occidentale - è questo: un « sistema protettivo » delle libertà individuali. Non sarà, questo, un ideale entusiasmante per i bambini viziati del nostro tempo. Ma è l'ideale che vanno riscoprendo coloro che hanno la malasorte di vivere alla mercé dei despoti. E quali che siano gli altri elementi o attributi della democrazia che ci sono cari, l'elemento irrinunziabile, il sine qua non della democrazia, è questo suo elemento garantistico. Difatti il «messaggio » potrà essere neutralizzato, e l'《arma simbolica » potrà essere tenuta sotto controllo, solo a questa condizione: che la democrazia come sistema protettivo sopravviva, e anzi venga rafforzata. Altrimenti la profezia è agevole: l'alternativa è una perfetta tirannide, perfezionata, appunto, dalla tecnologia dell'uomo. La contro-utopia di Orwell, 1984, con il suo linguaggio sterilizzato, e con il suo occhio televisivo che scruta ogni angolo e ogni momento della nostra esistenza, costituisce una raffigurazione perfettamente plausibile di dove può approdare un « controllo simbolico » totalitario o totalitaristico. $\mathrm{Da}$ un punto di vista tecnico, nulla osta. Il quesito è politico, e cioè investe le condizioni politiche che portano, o possono portare, dalle nostre democrazie populistiche e onni-permissive, al regime del Grande Fratello.

Intendiamoci sulla vera difficoltà delle profezie politiche. La previsione politologica è estremamente difficile sul versante costruttivo, o diciamo pure ingegneristico. $\mathrm{Ma}$ se la pars construens del discorso richiede davvero visione e genialità profetica, la pars destruens della prognosi richiede soltanto una ordinata « analisi delle condizioni $»$. Detto in parole piane, non è difficile prevedere le « crisi ». Si può sbagliare, e di parecchio, sui tempi, su quando una crisi di regime o di civiltà verrà a maturazione. $\mathrm{Ma}$ da quando

sottolinea le caratteristiche di altissima artificialità, precisione e fragilità della Città fondata sulla tecnologia elettronica. 
il mondo è mondo le crisi sono state previste, e sono esplose per le ragioni preannunziate, e cioè per quelle ragioni di cui gli storici hanno poi riconosciuto, ex post, la validità. Cosí come Tocqueville ha previsto a suo tempo l'interna meccanica di sviluppo dei sistemi democratici, analogamente si può prevedere, oggi, se la democrazia di cui Tocqueville ha visto la nascita e predetto il decorso abbia o non abbia un futuro. In verità, precalcolare le possibilità di sopravvivenza della democrazia (nella sua condizione sine qua non di sistema protettivo) non presenta difficoltà intrinseche maggiori del precalcolare le condizioni di agibilità di una megalopoli tecnotronica di 10 milioni di abitanti. La differenza sta soprattutto nel fatto che la previsione politica è assai meno in auge della proiezione tecnologica. E vale capire come mai.

Un primo motivo è la novità della previsione tecnologica, che la rende per ciò stesso un futuribile piú esaltante di quelli battuti da tempo. Senza contare che la velocità del progresso e dell'invenzione tecnologica offre ad ogni momento ulteriori elementi di novità. Un altro ordine di motivi è che la «scientificizzazione » dello studio della politica non avviene senza perdite, una delle quali è l'atrofia della immaginazione politologica. Vi è poi, come già accennavo, la differenza tra proiezioni da dati soffici ed estrapolazioni da dati duri, tangibili e misurabili. Tutti questi motivi non tolgono che qualsiasi previsione a lunga scadenza è viziata da irrealismo se non mette in conto $\mathrm{i}$ 《 futuri possibili » della politica.

Prendiamo la previsione di Bell secondo la quale la classe dominante del futuro sarà una classe tecnologica forte del suo sapere teoretico: una classe che può essere chiamata - seguendo Shonfield - la «classe teoretica $»^{4}$. Una classe teoretica qualificata dalla concomitante profezia di Bell secondo la quale le «istituzioni dominanti » del futuro saranno le istituzioni intellettuali nelle quali si sviluppa e trasmette il sapere scientifico: università, laboratori scientifici, centri di ricerca, e simili. Orbene, è a questo punto che non riesco piú a seguirlo. Perché è a questo punto che Bell - e, in generale, le previsioni a fondamento socio-economico e tecno-scientifico - dimenticano, o scontano troppo facilmente, la politica.

4 Piú esattamente la società post-industriale di Bell è caratterizzata lungo cinque dimensioni dai seguenti attributi: 1) nel settore economico il passaggio dalla società produttiva di beni alla società dei servizi; 2) nel settore occupativo il prevalere di una classe professionale e tecnica; 3) in sede tecnologica l'affermarsi di una nuova tecnologia intellettuale; 4) nella dimensione del cambiamento una crescita tecnica che si autoalimenta; 5) nell'insieme, la centralità del sapere teoretico. Come si vede, ben tre dimensioni su cinque $\left(1 \mathrm{a} 2^{\mathrm{a}}, 3^{\mathrm{a}}\right.$, e $\left.5^{\mathrm{a}}\right)$ concorrono a configurare l'idea di « classe teoretica». 
Politici, intellettuali e tecnocrati.

In essenza il problema è come il sapere (chi sa) si rapporta o meno con il potere (chi comanda). Le combinazioni possibili sono quattro:

1) Potere senza sapere.

2) Sapere senza potere.

3) I sapienti hanno anche il potere.

4) I potenti hanno anche il sapere.

Sino all'avvento della società industriale tra classe politica e classe teoretica gli incontri sono stati sporadici, i connubi marginali. I potenti poco o nulla sapevano, e $\mathrm{i}$ sapienti poco o nulla potevano. Questa separazione viene meno con l'avvento della società tecnologica e ancor piú della società tecnotronica. Il che comporta che il potere è moltiplicato dal sapere o, viceversa, che il sapere è corredato di potere. Ma i casi sono diversi: governo della scienza (ipotesi 3), oppure governo mediante la scienza (ipotesi 4)? La teoria della «classe teoretica 》 prevede che saranno i sapienti ad esercitare il potere. E, sotto nuove ed aggiornate vesti, la vecchia illusione platonica del filosofo-re. Dico illusione perché ritengo che tale sia destinata a restare. Anche se governassero gli scienziati, resta da dimostrare che governerebbero come scienziati. In ogni caso il potere resterà nelle mani degli «specialisti del potere », di coloro che fanno dell'acquisto e dell'esercizio del potere l'obiettivo primario, se non esclusivo, della propria esistenza. Altrimenti dicendo, la nozione di potere rinvia all'esistenza di una classe potestativa per antonomasia, tale perché detiene ed esercita quel potere che è sovraordinato ad ogni altro, e che viene identificato come potere «politico », come il potere di comandare sulla città nel suo insieme. Orbene, nella misura in cui gli intellettuali sono intellettuali e gli scienziati sono uomini di scienza, nella stessa misura non si qualificano come classe potestativa «sovrana », e cioè politica. Una classe teoretica ha potere nel proprio àmbito, che è quello delle istituzioni scientifiche, e non nell'àmbito delle istituzioni politiche.

La mia previsione è, dunque, che anche nella società tecnologica piú avanzata il governo resterà un governo dei politici, anche se diventerà sempre piú un governo orientato e rinforzato dagli esperti. Il che precisa la quarta combinazione, o ipotesi, di cui sopra. Non è che, alla lettera, $\mathrm{i}$ potenti siano anche sapienti. È che $\mathrm{i}$ detentori del potere politico si avvalgono di coloro che sanno come di una necessaria e addizionale risorsa del loro potere. Il braccio secolare si rinforza con il braccio intellettuale. Di fatto è cosí che il politico moderno, alla Kennedy o alla Servain-Schreiber, percepisce e fruisce della classe teoretica: come di una risorsa strategica.

Occorre dunque intendersi bene su come governo e scienza si 
possono combinare. Nella versione e visione tecnocratica l'argomento è. che nella misura in cui la classe potestativa ha bisogno della classe teoretica, nella stessa misura è condizionata da quest'ultima e deve spartire con essa il proprio potere. Questa previsione si è avverata nell'ambito industriale, e nella misura in cui il potere è un «potere proprietario». Ma la cosiddetta rivoluzione manageriale non è che un caso di applicazione del principio secondo il quale il potere non consiste tanto nella titolarità quanto nell'esercizio. Il manager della grande industria o della corporazione gigante è chi esercita un potere la cui titolarità si è polverizzata, o che difetta di un titolare tecnicamente idoneo. $\mathrm{Ma}$ il caso del potere politico è del tutto diverso. Il potere politico non è una proprietà, una « cosa che si possiede », e da gran tempo non necessita di basi patrimoniali. Il potere politico è un «potere relazionale » che appartiene a chi lo esercita. L'argomento che vale per il rapporto tra managers e proprietari non vale per il rapporto tra scienziati e politici. Pertanto l'aspetto rilevante del governo mediante la scienza non sta nella traslazione di potere - che è modesta - ma nella fantastica moltiplicazione delle potenzialità del potere. Un potere senza sapere è un potere limitato e circoscritto dal proprio difetto di cognizioni. $\mathrm{Ma}$ un potere assistito dal sapere - e da quel sapere tecnologico che si risolve in una tecnologia del controllo sull'uomo - diventa eo ipso un potere potenzialmente illimitato. A meno che non venga limitato altrimenti.

Si dirà che questa interpretazione è pessimistica, e che la mia previsione sottintende che gli intellettuali si presteranno a «servire ». In effetti è cosí. Non vedo perché la nuova «classe teoretica » debba differire per questo rispetto dagli intellettuali di tutti i tempi. L'intellettuale vive su margini sottili, difetta di indipendenza economica, opera in serre $o$ in torri d'avorio. Salvo nobilissime ma numericamente esigue eccezioni, non ha la vocazione del guerriero né il temperamento del combattente. L'intellettuale protesta, attacca il potere e magari sale sulle barricate nelle società vellutate, quando governano (direbbe Pareto) le «volpi », e cioè quando il ribellismo non è un gran rischio, e anzi rischia di rendere. $\mathrm{Ma}$ nelle società che Pareto direbbe governate dai «leoni », per dieci che resistono novanta cedono. Al paragone, i lavoratori mentali sono piú malleabili dei lavoratori manuali: la loro mente è agile, lavora in fretta, e trova lestamente modi e motivi per adattarsi. Sotto i cieli di tutte le tirannidi la vicenda del tradimento dei chierici si ripete. $E$ non si tratta veramente, o necessariamente, di «tradimento». L'umanista che ripudia oggi $i$ valori che aveva professato sino a ieri, può essere tacciato di tradimento; ma una classe teoretica di stampo tecnico-scientifico procede nel suo lavoro con chi le fornisce gli strumenti per lavorare. E quindi sarebbe un errore puntare - al cospetto del Grande Fratello - su una scienza 
che rifiuta di collaborare, che non diventa instrumentum regni. E ben possibile che le istituzioni intellettuali diventino dominanti; ma è altrettanto possibile che gli intellettuali diventino, al tempo stesso, dominati.

La proiezione tecnologica dà piú o meno per scontato che le presenti condizioni politiche - una società aperta, pluralistica, tollerante - siano destinate a rimanere press'a poco eguali. La clausola tacita delle profezie tecno-scientifiche è: a parità di condizioni politiche. Ma ogni giorno che passa conferma quanto questa clausola sia poco plausibile. Lasciamo stare la contro-cultura. Resta il fatto che una «statica politica » è ben poco verosimile in un mondo in cui tutto cambia ad una velocità traumatizzante. Donde la mia preoccupazione di mettere in evidenza quanto un babitat tecnologico aggiunge, quantomeno in potenziale, al potere del potere. Non lasciamoci ingannare e addormentare dal vuoto di potere che caratterizza, al momento, il mondo occidentale. Il fatto è che con la «tecnologia dell'uomo» il potere di alcuni uomini su altri uomini acquista - se scatenato - proporzioni, o meglio sproporzioni, di crescita esponenziale. Il che rende urgente, mi sembra, un massiccio ritorno di attenzione dalla previsione tecnologica alla previsione politica. Le profezie - è noto - si autodistruggono in virtú di un «effetto riflettente », e cioè perché il futuro rimbalza sul presente. È cosí che anche il mero prevedere aiuta a provvedere.

Bell si chiede se la politica debba essere intesa come una fonte autonoma, o addirittura la fonte ultima, irriducibile, di differenziazione e conflitti tra le società. Direi senz'altro di sí: se non altro perché in ultima istanza la politica si alimenta, e viene alimentata da un "gioco contro persone ». Beninteso, il gioco contro persone è parte della vita di tutti, in tutte le sfere. Ma la politica è $i l$ gioco contro persone per eccellenza: la posta è tutta lí. Nella « vincita » (vincita di potere) il politico non trova solo la sua massima gratificazione, ma la sua stessa condizione di sopravvivenza (come politico). Non mi pare dubbio, dunque, che il gioco del potere costituisca una fonte indipendente, e che si auto-alimenta, di diversificazione e di antagonismo tra le società umane. La politica resterà. $\mathrm{Ma}$ non è affatto detto che resti quell'animale domestico che è stato domato dai sistemi protettivi. Come «neutralizzare» il potere della politica nella società post-industriale o tecnotronica, è davvero motivo di suprema preoccupazione. Stranamente, se non assurdamente, non diamo gran mostra di occuparcene. 